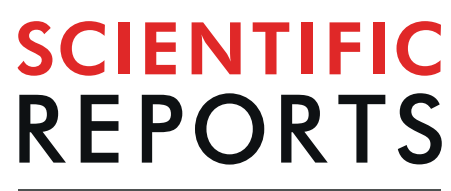

\title{
Factors associated with habitual time spent in different physical activity intensities using multiday accelerometry
}

\author{
Lina Jaeschke ${ }^{1 *}$, Astrid Steinbrecher ${ }^{1}$, Heiner Boeing ${ }^{2}$, Sylvia Gastell ${ }^{2}$, Wolfgang Ahrens $\mathbb{D}^{3,4}$, \\ Klaus Berger ${ }^{5}$, Hermann Brenner ${ }^{6}$, Nina Ebert ${ }^{7}$, Beate Fischer ${ }^{8}$, Karin Halina Greiser ${ }^{9}$, \\ Wolfgang Hoffmann ${ }^{10}$, Karl-Heinz Jöckel ${ }^{11}$, Rudolf Kaaks ${ }^{9}$, Thomas Keil ${ }^{12}$, Yvonne Kemmling ${ }^{13}$, \\ Alexander Kluttig ${ }^{14}$, Lilian Krist ${ }^{12}$, Michael Leitzmann ${ }^{8}$, Wolfgang Lieb ${ }^{15}$, Jakob Linseisen ${ }^{16,17}$, \\ Markus Löffler ${ }^{18}$, Karin B. Michels ${ }^{19}$, Nadia Obi ${ }^{20}$, Annette Peters ${ }^{21}$, Sabine Schipf ${ }^{22}$, \\ Börge Schmidt ${ }^{11}$, Melanie Zinkhan ${ }^{14}$ \& Tobias Pischon ${ }^{1,23,24,25}$
}

To investigate factors associated with time in physical activity intensities, we assessed physical activity of 249 men and women (mean age 51.3 years) by 7 -day $24 \mathrm{~h}$-accelerometry (ActiGraph GT3X+). Triaxial vector magnitude counts/minute were extracted to determine time in inactivity, in low-intensity, moderate, and vigorous-to-very-vigorous activity. Cross-sectional associations with sex, age, body mass index, waist circumference, smoking, alcohol consumption, education, employment, income, marital status, diabetes, and dyslipidaemia were investigated in multivariable regression analyses. Higher age was associated with more time in low-intensity (mean difference, $7.3 \mathrm{~min} / \mathrm{d}$ per 5 years; $95 \%$ confidence interval $2.0,12.7)$ and less time in vigorous-to-very-vigorous activity $(-0.8 \mathrm{~min} / \mathrm{d} ;-1.4$, $-0.2)$, while higher BMI was related to less time in low-intensity activity $(-3.7 \mathrm{~min} / \mathrm{d} ;-6.3,-1.2)$. Current versus never smoking was associated with more time in low-intensity $(29.2 \mathrm{~min} / \mathrm{d} ; 7.5,50.9)$ and less time in vigorous-to-very-vigorous activity $(-3.9 \mathrm{~min} / \mathrm{d} ;-6.3,-1.5)$. Finally, having versus

\footnotetext{
${ }^{1}$ Molecular Epidemiology Group, Max Delbrück Center for Molecular Medicine in the Helmholtz Association (MDC), Berlin, Germany. ${ }^{2}$ Division of Epidemiology, German Institute of Human Nutrition Potsdam-Rehbruecke, PotsdamRehbruecke, Germany. ${ }^{3}$ Leibniz Institute for Prevention Research and Epidemiology - BIPS, Bremen, Germany. ${ }^{4}$ Institute of Statistics, Faculty of Mathematics and Computer Science, University of Bremen, Bremen, Germany. ${ }^{5}$ Institute of Epidemiology and Social Medicine, University of Münster, Münster, Germany. ${ }^{6}$ Division of Clinical Epidemiology and Aging Research, German Cancer Research Center, INF 581, Heidelberg, Germany. ${ }^{7}$ German Diabetes Center (DDZ), Leibniz Center for Diabetes Research, Heinrich Heine University, Institute for Biometrics and Epidemiology, Düsseldorf, Germany. ${ }^{8}$ Department of Epidemiology and Preventive Medicine, University of Regensburg, Regensburg, Germany. ${ }^{9}$ German Cancer Research Center (DKFZ), Heidelberg, Germany. ${ }^{10}$ Section Epidemiology of Health Care and Community Health, Institute for Community Medicine, University Medicine Greifswald, Greifswald, Germany. ${ }^{11}$ Institute for Medical Informatics, Biometry and Epidemiology (IMIBE), University Hospital Essen, University of Duisburg-Essen, Essen, Germany. ${ }^{12}$ Institute for Social Medicine, Epidemiology and Health Economics, Charité - Universitätsmedizin Berlin, Berlin, Germany. ${ }^{13}$ Department of Epidemiology, Helmholtz-Centre for Infection Research, Braunschweig, Germany. ${ }^{14}$ Institute of Medical Epidemiology, Biometry and Informatics, Martin-Luther-University, Halle (Saale), Germany. ${ }^{15}$ Institute of Epidemiology, Kiel University, Kiel, Germany. ${ }^{16}$ Chair of Epidemiology, LMU Munich at UNIKA-T, Augsburg, Germany. ${ }^{17} \mathrm{Helmholtz} \mathrm{Zentrum} \mathrm{München,}$ IRG Clinical Epidemiology, Neuherberg, Germany. ${ }^{18}$ Institute for Medical Informatics, Statistics and Epidemiology, Leipzig, Germany. ${ }^{19}$ Institute for Prevention and Cancer Epidemiology, Faculty of Medicine and Medical Center, University of Freiburg, Freiburg, Germany. ${ }^{20}$ Institute for Medical Biometry and Epidemiology, University Medical Center Hamburg-Eppendorf, Hamburg, Germany. ${ }^{21}$ Institute of Epidemiology, Helmholtz Zentrum München German Center for Health and Environment, Neuherberg, Germany. ${ }^{22}$ Institute for Community Medicine, University Medicine Greifswald, Greifswald, Germany. ${ }^{23}$ Charité - Universitätsmedizin Berlin, Berlin, Germany. ${ }^{24}$ German Center for Cardiovascular Research (DZHK), partner site Berlin, Berlin, Germany. ${ }^{25}$ MDC/BIH Biobank, Max Delbrück Center for Molecular Medicine and Berlin Institute of Health, Berlin, Germany. *email: lina.jaeschke@mdc-berlin.de
} 
not having a university entrance qualification and being not versus full time employed were associated with more inactivity time ( $35.9 \mathrm{~min} / \mathrm{d} ; \mathbf{1 3 . 0}, 58.8$, and $66.2 \mathrm{~min} / \mathrm{d} ; 34.7,97.7$, respectively) and less time in low-intensity activity $(-31.7 \mathrm{~min} / \mathrm{d} ;-49.9,-13.4$, and $-50.7 ;-76.6,-24.8$, respectively). The assessed factors show distinct associations with activity intensities, providing targets for public health measures aiming to increase activity.

Habitual physical activity (PA) is crucial in the aetiology of noncommunicable diseases ${ }^{1}$. Insufficient PA is one of the leading risk factors for mortality and disability-adjusted life years globally ${ }^{1,2}$. For adults, the World Health Organization (WHO) recommends at least 150 minutes of moderate or 75 minutes of vigorous PA per week or metabolic equivalents ${ }^{1}$. Previous studies have shown that meeting these criteria reduces morbidity and mortality risk $^{3,4}$.

Knowing factors associated with PA is important, since modifiable factors might represent targets for public health measures that aim to increase PA, while unmodifiable factors might allow identification of physically active and inactive target subgroups ${ }^{5}$. Sex, age, anthropometry, health, smoking, alcohol consumption, marital status, education, employment, and income are important factors from different domains that may be related to PA, including biology, behaviour, socio-culture, and socio-economy. However, evidence on a relationship to PA is inconsistent for most of these factors and mainly based on self-reported $\mathrm{PA}^{6-13}$, being prone to measurement error, especially regarding PA intensities ${ }^{14}$. Assessing both PA duration and intensity is important in epidemiologic studies, as both are independent behaviours and risk factors ${ }^{3,15}$. Accelerometers detect accelerations of the body under free-living conditions, capturing PA duration and intensity more precisely than self-reports. However, previous studies largely rely on uniaxial accelerometry applied during waking only ${ }^{16-19}$, while new cohorts, like the German National Cohort (NAKO Gesundheitsstudie) or the UK Biobank use triaxial $24 \mathrm{~h}$-accelerometry ${ }^{20-22}$. Further, while convincing or probable evidence from systematic analyses on PA-related factors in general is scarce ${ }^{6-11,13,23,24}$, to our knowledge, it is lacking for $24 \mathrm{~h}$-accelerometry-based PA intensities in the adult German population.

The aim of this study was to investigate factors associated with habitual time spent in different activity intensities, and to estimate the likelihood of meeting the WHO PA recommendation in a sample from the general German adult population using multiday $24 \mathrm{~h}$-accelerometry.

\section{Materials and Methods}

Pretest of the German National Cohort (NAKO Gesundheitsstudie). Data were collected as part of a pretest of the German National Cohort (NAKO Gesundheitsstudie) ${ }^{20}$. The NAKO is an ongoing population-based cohort study that recruited 205,000 adults aged 20-69 years from the general population in 18 study centres distributed across Germany, in order to study causes and mechanisms of major chronic diseases ${ }^{20,21}$. Pretests were conducted upfront with the primary aim to test and implement infrastructures and study protocols ${ }^{25}$. Data used in the present analyses were collected during pretest 2 between May 2012 and April 2013. Each study centre was expected to draw an age- and sex-stratified random sample from local municipal population registries ( $50 \%$ men, 10 -years age groups from $20-69$ years, with $10 \%$ of participants in each of the two younger age groups, and $26.6 \%$ of participants in each of the other age groups) to examine at least 100 participants according to a common proto$\mathrm{col}^{20,25}$. Inclusion criteria were residence in the catchment area of the municipal registries covered by the respective study centre, age 20-69 years, proficiency of the German language, and ability to provide informed consent.

The study protocol of the NAKO pretest was approved by the ethics committee of the Bayerische Landesärztekammer (study center: Augsburg), the Charité - Universitätsmedizin Berlin (Berlin-Center, Berlin-North, Berlin-South/Brandenburg), the Ärztekammer Niedersachsen (Hannover/Braunschweig), the Ärztekammer Bremen (Bremen), the Medical Faculty of the Heinrich-Heine-Universität Düsseldorf (Düsseldorf), the Albert-Ludwigs-Universität Freiburg (Freiburg), the Medical Faculty of the Martin-Luther-Universität Halle-Wittenberg (Halle), the Ärztekammer Hamburg (Hamburg), the Medical Faculty Heidelberg (Heidelberg), the Medical Faculty of the Christian-Albrechts-Universität Kiel (Kiel), the Ärztekammer Westfalen-Lippe and the Medical Faculty of the Westfälische Wilhelms-Universität (Münster), the Universitätsmedizin Greifswald (Neubrandenburg), the Medical Faculty of the Universität Regensburg (Regensburg), and the Ärztekammer des Saarlandes (Saarbrücken), as well as by the local data protection officers. All investigations were carried out in accordance with the relevant guidelines and regulations, and written informed consent was obtained from all participants before inclusion ${ }^{20}$.

Data collection. Data collection followed a standardized protocol across study centres. Participants visited the study centre and performed a personal computer-assisted interview on age, smoking status, alcohol consumption, highest level of education, employment status, net household income, marital status, and history of diabetes mellitus and dyslipidaemia ${ }^{20,21}$. Participants underwent an anthropometric measurement by trained personnel according to WHO guidelines ${ }^{21}$. Body height $(\mathrm{cm})$ was measured using the stadiometer SECA 285 (Hamburg, Germany) or an equivalent model, body weight $(\mathrm{kg})$ using the bioelectrical impedance analysis device SECA mBCA 515 (Hamburg, Germany) or an equivalent model, and waist circumference (WC, $\mathrm{cm}$ ) using the measuring tape SECA 201 (Hamburg, Germany). Body mass index (BMI) was calculated as body weight divided by the square of body height $\left(\mathrm{kg} / \mathrm{m}^{2}\right)$. All measurements were performed with an accuracy of one decimal place ${ }^{21}$.

PA was assessed by the triaxial accelerometer GT3X+ (ActiGraph LLC, Pensacola, FL, USA). Participants were asked to continuously wear the accelerometer $24 \mathrm{~h} / \mathrm{d}$ on the right hip for one week and to send it back by mail thereafter. They were instructed to take off the device for high contact sports, sauna sessions, and water-based activities $>30$ minutes only ${ }^{21}$. ActiLife (ActiGraph LLC, Pensacola, FL, USA) software was used to process the acceleration data (initialization and downloading: versions 6.4.3/5, 6.5.2/3, and 6.7.3; data processing: versions 


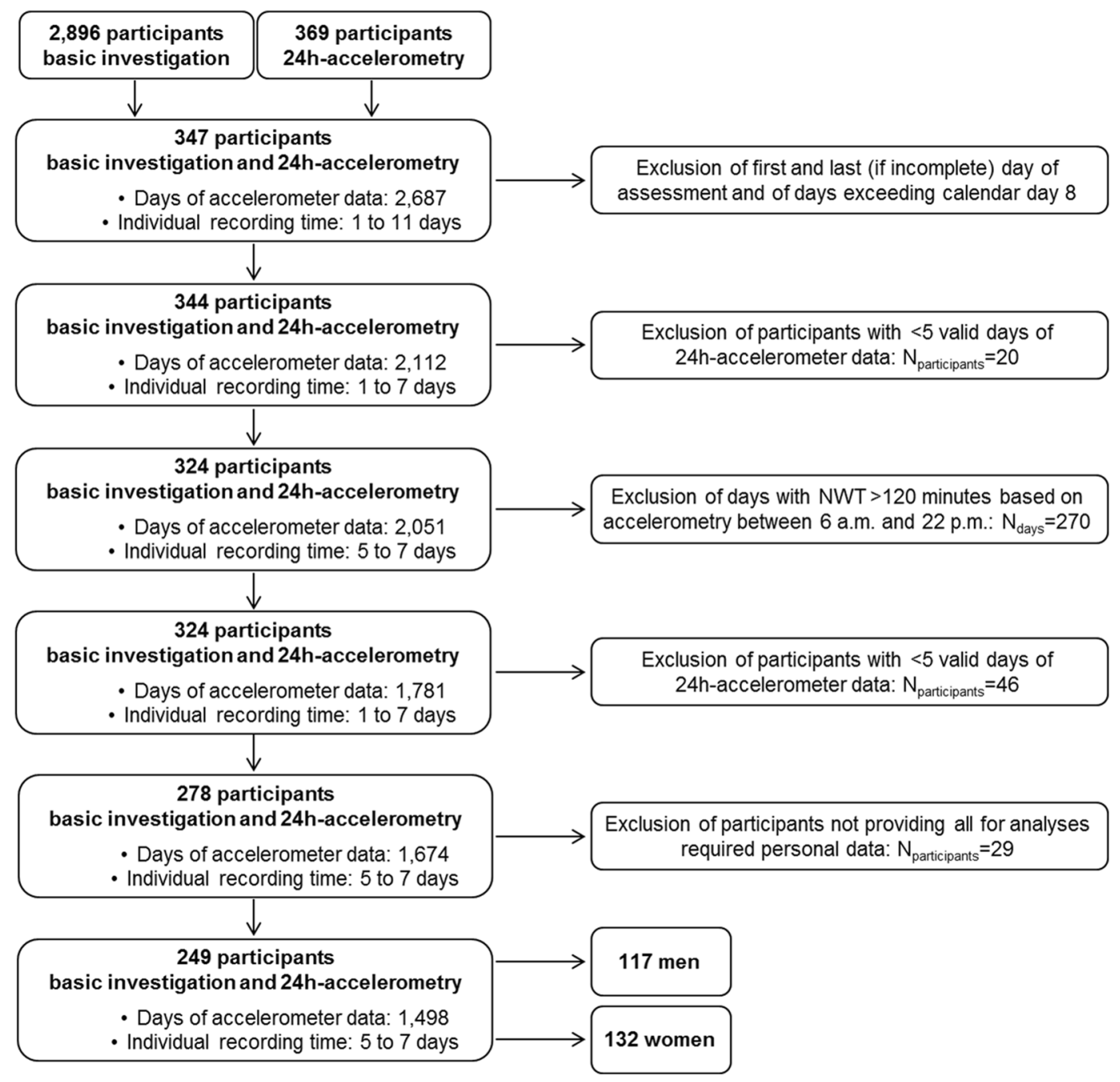

Figure 1. Selection of the study population from pretest 2 of the German National Cohort (NAKO Gesundheitsstudie). Initially, in pretest 2 of the German National Cohort (NAKO Gesundheitsstudie), 2,896 participants provided data on basic characteristics (interview and anthropometry), 369 participants provided data on 24 h-accelerometry (GT3X+, ActiGraph LLC, Pensacola, FL, USA), and 347 participants provided data on both. Finally, 249 participants fulfilled all inclusion criteria and were included for the present analyses on factors potentially associated with $24 \mathrm{~h}$-accelerometry-based physical activity intensities. NWT, non-wear time.

6.12.1 to 6.13.2). Raw triaxial acceleration data were sampled by a 12 bit analogue-to-digital converter (dynamic range, $\pm 6 \mathrm{G}$ ) using a constant $100 \mathrm{~Hz}$ sampling rate (stored at $100 \mathrm{~Hz}$ rate), with the filter set to 'normal' (default) and the 'Idle Sleep Mode' being disabled. Raw triaxial acceleration data were converted into 10-seconds epochs on site and were transferred to a central data management facility. Each study centre was supposed to apply $24 \mathrm{~h}$-accelerometry in at least 20 participants.

Study sample. In total 2,896 participants were included in pretest 2 (response, $18.7 \%$ ). Of these, 347 took part in 24 h-accelerometry (Fig. 1). From these, we excluded the first day of assessment (study centre visit) and all days exceeding day 8. Finally, the last day of assessment was excluded when incomplete. For final inclusion, a data set needed to encompass $\geq 5$ days not showing any accelerometer non-wear time $>120$ minutes during a presumed waking period between $6 \mathrm{AM}$ and $10 \mathrm{PM}$ automatically detected by ActiLife. These decisions were based on our previous studies, showing (1) that having $\geq 5$ days of $24 \mathrm{~h}$-accelerometry data available provides sufficient validity for PA assessment (expected correlation between observed and true PA parameter $\geq 0.85$ ) and (2) that automated non-wear time detection is most specific and sensitive when using a 120-min algorithm ${ }^{26,27}$. We further excluded participants with missing information on any variables included in the multivariable model. In total, 98 participants were excluded, resulting in 249 participants (117 men, 132 women) being finally included in the present analyses.

Operationalization of physical activity intensities. For each participant and day, the triaxial 'vector magnitude counts per minute' (cpm) was used to determine PA parameters based on the triaxial-derived algorithm 'Freedson Adult VM3 (2011)' implemented in ActiLife. Referring to metabolic equivalents of tasks (METs), 
this algorithm classifies 0-2,690 cpm as light (equivalent to <3.0 METs; e.g., slow walking), 2,691-6,166 cpm as moderate (3.0-5.99 METs; e.g., gymnastics), 6,167-9,642 cpm as vigorous (6.0-8.99 METs; e.g., jogging), and $\geq 9,643 \mathrm{cpm}$ as very vigorous activity ( $\geq 9.0 \mathrm{METs}$; e.g., carrying loads upstairs, squash $)^{28}$. As reported previously, we further subdivided 'light' activity into time in 'inactivity' $(0-78 \mathrm{cpm})$ and in 'low-intensity' activity $(79-2,690 \mathrm{cpm})^{27}$. Since mean daily time in very vigorous activity was short (geometric mean, GM, $0.16 \mathrm{~min} / \mathrm{d}$ ), we summed it with vigorous activity to vigorous-to-very-vigorous (VV) activity. Times spent in activity intensities were determined as mean $\mathrm{min} / \mathrm{d}$.

Additionally, we characterized participants according to WHO PA recommendations, which requires time in moderate or vigorous activity to be spent in bouts of $\geq 10$ minutes $^{1}$. For each participant, we determined the total time spent in 10-minutes bouts in moderate or VV activity, divided the sum by the number of assessment days, and multiplied by 7 to obtain mean week estimates. Estimates $\geq 150$ minutes of moderate or $\geq 75$ minutes of VV activity per week were classified as 'meeting WHO recommendation'. Otherwise, we determined if participants achieved a 'metabolic equivalent' of these, as mentioned, although not defined, by the $\mathrm{WHO}^{1}$. Fulfilling the WHO criteria is equivalent to achieving $450 \mathrm{METs} / \mathrm{week}$, when using 3 and 6 METs as limits for moderate and vigorous activity, respectively ${ }^{1}$. To acknowledge higher activity intensities within both intensity levels, we multiplied the weekly bouts estimates in moderate and VV activity by 4 and $8 \mathrm{METs}$, respectively ${ }^{29}$. If participants achieved $\geq 450 \mathrm{METs}$ /week based on moderate and VV activity, this was classified as 'meeting WHO recommendation'. Participants not meeting any of the aforementioned criteria were classified as 'not meeting WHO recommendation'.

Statistical analyses. To investigate factors associated with time spent in inactivity and in low-intensity, moderate, and VV activity, we used four separate linear regression models with robust variance estimation ${ }^{30}$, each of the PA measures as single outcome, and the following independent variables: sex (male, female), age (continuously), BMI (continuously), WC (continuously, residually adjusted for BMI), smoking status (never, current, or former), alcohol consumption (never, $\leq 1 \times /$ month, $2-4 \times /$ month, $2-3 \times /$ week, or $\geq 4 \times /$ week), university entrance qualification (yes, no), employment status (full time, part time or not employed (including retired)), net household income/month $(<2,500 €, 2,500-3,999 €, \geq 4,000 €$, or not available), marital status (married, not married), diabetes mellitus (yes, no), dyslipidaemia (yes, no), and study centre (dummy variables). We tested for sex differences by further including interaction terms with sex for each independent variable, and we performed analyses stratified by sex.

To investigate factors related to meet the WHO PA recommendation (yes, no), we used logistic regression analyses and the same respective models as conducted for the linear analyses.

In sensitivity analyses, all analyses were repeated using a sample that was selected by using a more conservative 60-minutes instead of 120-minutes non-wear time algorithm, resulting in 201 participants (86 men, 115 women) and a total of 1,070 24h-accelerometry days. In a further analysis, we determined PA intensities using common uniaxial-derived cut points proposed by Freedson et al., defining 0-99 cpm measured by the vertical axis as 'sedentary', $100-1,951 \mathrm{cpm}$ as 'light', $1,952-5724 \mathrm{cpm}$ as 'moderate', and $\geq 5,725 \mathrm{cpm}$ as VV PA ${ }^{31}$ and repeated linear analyses. Finally, since $76 \%$ of persons in the group 'not employed' were retired, we repeated linear analyses by replacing 'employment status' by 'retired, yes vs. no' and 'not employed, yes vs. no' (yes: including jobless persons, persons permanently unable to work, and housewives/househusbands).

To test for differences between included and excluded participants, unpaired t-tests or Mann-Whitney U tests, respectively, were used for continuous variables, and Chi-Square tests were performed for discrete variables.

P-values presented are two-tailed, with $\mathrm{p}<0.05$ considered statistically significant. To account for multiple testing using Bonferroni adjustments, $\mathrm{p}$-values $<0.01$ in the linear and logistic regression analyses (four and one regression models, respectively) and $<0.0009$ in the sex difference analyses ( 11 interaction terms included in each of the five regression models) were considered statistically significant. Analyses were performed using SAS ${ }^{\circledR}$ Enterprise Guide $^{\circledR}$ (version 7.1; SAS Institute Inc., Cary, NC).

\section{Results}

In total, 249 participants (47.0\% men) were included (Table 1, and Supplementary Table S1). Compared to participants excluded from analyses $(n=98)$, participants included were more likely to have a net household income $>4,000 € /$ month $(23.7 \%$ versus $10.2 \%)$ and higher WC $(90.6 \mathrm{~cm}$ versus $87.4 \mathrm{~cm})$; otherwise, basic characteristics were similar (data not shown).

Most participants provided 24h-accelerometry data for 6 observation days (53.4\%; 5 days: $22.5 \%$; 7 days: $24.1 \%$; Table 2). Men spent more time in inactivity and less time in low-intensity activity than women $(\mathrm{p}=0.02$ and $\mathrm{p}=0.002$, respectively). About one third of participants met the WHO PA recommendation, with $13.3 \%$ and $7.6 \%$ fulfilling the criteria of $150 \mathrm{~min} /$ week moderate and $75 \mathrm{~min} /$ week VV activity, respectively, spent in bouts of at least 10 minutes, and $11.2 \%$ meeting the metabolic equivalent. Men were slightly less likely to meet the criteria than women $(\mathrm{p}=0.04)$.

Linear regression analyses. Regarding the multivariable-adjusted associations of investigated factors with time spent in different PA intensities, higher age was significantly associated with more time spent in low-intensity $(7.3 \mathrm{~min} / \mathrm{d}$ per 5 years; $95 \%$ confidence interval, CI, 2.0 to 12.7$)$ but less time in VV activity $(-0.8 \mathrm{~min} / \mathrm{d} ;-1.4$ to -0.2) (Table 3). Furthermore, a 1-unit higher BMI was significantly associated with less time in low-intensity activity $(-3.7 \mathrm{~min} / \mathrm{d},-6.3$ to -1.2$)$. Additionally, current as compared to never smoking was significantly associated with more time in low-intensity $(29.2 \mathrm{~min} / \mathrm{d} ; 7.5$ to 50.9$)$ but less time in VV activity $(-3.9 \mathrm{~min} / \mathrm{d} ;-6.3$ to -1.5). Having a university entrance qualification as compared to no such qualification was significantly associated with more inactivity time $(35.9 \mathrm{~min} / \mathrm{d} ; 13.0$ to 58.8$)$ and less time spent in low-intensity activity $(-31.7 \mathrm{~min} / \mathrm{d}$; -49.9 to -13.4$)$. Finally, not versus full time employed persons spent more time in inactivity $(66.2 \mathrm{~min} / \mathrm{d} ; 34.7$ to 


\begin{tabular}{|c|c|c|c|c|c|c|}
\hline & \multicolumn{2}{|c|}{ Total $(\mathrm{N}=249)$} & \multicolumn{2}{|c|}{ Men $(n=117)$} & \multicolumn{2}{|c|}{ Women $(n=132)$} \\
\hline & mean & SD & mean & SD & mean & SD \\
\hline age, years & 51.3 & 10.8 & 50.9 & 11.1 & 51.6 & 10.7 \\
\hline height, $\mathrm{cm}$ & 171.0 & 9.5 & 178.2 & 6.8 & 164.6 & 6.6 \\
\hline weight, kg & 77.2 & 14.5 & 86.7 & 12.3 & 68.8 & 10.5 \\
\hline BMI, kg/m ${ }^{2}$ & 26.3 & 4.0 & 27.4 & 3.9 & 25.4 & 3.8 \\
\hline \multirow[t]{2}{*}{$\mathrm{WC}, \mathrm{cm}$} & 90.6 & 12.9 & 98.3 & 11.8 & 83.8 & 9.7 \\
\hline & n & $\%$ & n & $\%$ & n & $\%$ \\
\hline \multicolumn{7}{|l|}{ smoking status } \\
\hline never & 101 & 40.6 & 44 & 37.6 & 57 & 43.2 \\
\hline current & 58 & 23.3 & 26 & 22.2 & 32 & 24.2 \\
\hline former & 90 & 36.1 & 47 & 40.2 & 43 & 32.6 \\
\hline \multicolumn{7}{|l|}{ alcohol consumption } \\
\hline never & 7 & 2.8 & 3 & 2.6 & 4 & 3.0 \\
\hline max. $1 \times /$ month & 56 & 22.5 & 15 & 12.8 & 41 & 31.1 \\
\hline $2-4 \times /$ month & 74 & 29.7 & 31 & 26.5 & 43 & 32.6 \\
\hline $2-3 \times /$ week & 64 & 25.7 & 34 & 29.1 & 30 & 22.7 \\
\hline$\geq 4 \times /$ week & 48 & 19.3 & 34 & 29.1 & 14 & 10.6 \\
\hline \multicolumn{7}{|l|}{ school education } \\
\hline university entrance qualification & 120 & 48.2 & 65 & 55.6 & 55 & 41.7 \\
\hline no university entrance qualification & 129 & 51.8 & 52 & 44.4 & 77 & 58.3 \\
\hline \multicolumn{7}{|l|}{ employment status } \\
\hline full time & 135 & 54.2 & 85 & 72.6 & 50 & 37.9 \\
\hline part-time & 62 & 24.9 & 11 & 9.4 & 51 & 38.6 \\
\hline not employed & 52 & 20.9 & 21 & 17.9 & 31 & 23.5 \\
\hline \multicolumn{7}{|l|}{ net household income per month } \\
\hline$<2,500 €$ & 86 & 34.5 & 41 & 35.0 & 45 & 34.1 \\
\hline $2,500-4,000 €$ & 92 & 36.9 & 40 & 34.2 & 52 & 39.4 \\
\hline$>4,000 €$ & 59 & 23.7 & 33 & 28.2 & 26 & 19.7 \\
\hline n. a. & 12 & 4.8 & 3 & 2.6 & 9 & 6.8 \\
\hline \multicolumn{7}{|l|}{ marital status } \\
\hline married & 156 & 62.7 & 79 & 67.5 & 77 & 58.3 \\
\hline not married & 93 & 37.3 & 38 & 32.5 & 55 & 41.7 \\
\hline diabetes mellitus & 18 & 7.2 & 11 & 9.4 & 7 & 5.3 \\
\hline dyslipidaemia & 74 & 29.7 & 41 & 35.0 & 33 & 25.0 \\
\hline
\end{tabular}

Table 1. Basic characteristics of study participants, 2012-2013. Information was derived from self-reports during a personal interview, anthropometric measures were taken by trained personnel. BMI, body mass index; n, number; n. a., not available; SD, standard deviation; WC, waist circumference.

97.7) but less time in low-intensity activity $(-50.7 \mathrm{~min} / \mathrm{d} ;-76.6$ to -24.8$)$. When adjusted for multiple testing, sex differences in the multivariable-adjusted associations of investigated factors with time in activity intensities were not statistically significant (Supplementary Table S2).

Logistic regression analyses. Regarding the multivariable-adjusted associations of investigated factors with the likelihood of meeting the WHO PA recommendation, current compared to never smokers were substantially less likely to meet the criteria (odds ratio, OR; 95\% CI: $0.21 ; 0.08$ to 0.53 ) (Table 4). Sex differences in these associations were not statistically significant after accounting for multiple testing (Supplementary Table S3).

Sensitivity analyses. When repeating the analyses using the 60-minutes instead of 120 -minutes accelerometer non-wear time algorithm, results were quite similar to the main analyses, although the association of age, $\mathrm{BMI}$, and employment status with time in low-intensity activity were no longer statistically significant in these analyses (data not shown).

When evaluating the uniaxial accelerometry data, the association of age with time in light and VV activity, of BMI and smoking status with time in light activity, and of university entrance qualification with sedentary time were not statistically significant anymore compared to the equivalent triaxial-derived activity parameters (Supplementary Table S4).

Results for time in inactivity for persons being 'retired' and 'not employed due to other reasons' were similar as findings for the joint group of not employed persons (retired, yes vs. no: $68.0 \mathrm{~min} / \mathrm{d}$; 36.7, 99.3; not employed due to other reasons, yes vs. no: $65.0 \mathrm{~min} / \mathrm{d} ; 17.4,112.6$ ). 


\begin{tabular}{|c|c|c|c|c|c|c|}
\hline \multirow[b]{2}{*}{ PA parameter } & \multicolumn{2}{|c|}{ Total $(\mathrm{N}=249)$} & \multicolumn{2}{|c|}{ Men $(n=117)$} & \multicolumn{2}{|c|}{ Women $(n=132)$} \\
\hline & GM & 95\% CI & GM & $95 \% \mathrm{CI}$ & GM & 95\% CI \\
\hline time in inactivity, $\mathrm{min} / \mathrm{d}$ & 1010.6 & $(999.6,1021.8)$ & 1024.3 & $(1008.1,1040.8)$ & 998.7 & $(983.8,1013.8)$ \\
\hline time in low-intensity activity, $\mathrm{min} / \mathrm{d}$ & 327.0 & $(318.4,335.9)$ & 312.8 & $(301.0,325.0)$ & 340.2 & $(328.1,352.7)$ \\
\hline time in moderate activity, $\mathrm{min} / \mathrm{d}$ & 78.8 & $(75.0,82.9)$ & 79.5 & $(73.8,85.5)$ & 78.3 & $(73.0,83.9)$ \\
\hline time in VV activity, $\mathrm{min} / \mathrm{d}$ & 2.7 & $(2.3,3.2)$ & 3.0 & $(2.4,3.8)$ & 2.5 & $(2.00,3.1)$ \\
\hline 10-minutes bouts of activity & median & IQR & median & IQR & median & IQR \\
\hline time in bouts of moderate activity, min/week & 44.8 & $(12.3,100.0)$ & 35.0 & $(12.3,78.2)$ & 51.9 & $(12.4,120.7)$ \\
\hline time in bouts of VV activity, min/week & 0.0 & $(0.0,0.0)$ & 0.0 & $(0.0,0.0)$ & 0.0 & $(0.0,0.0)$ \\
\hline meeting WHO PA recommendation $^{\mathrm{a}}$ & $\mathbf{n}$ & $\%$ & $\mathbf{n}$ & $\%$ & n & $\%$ \\
\hline no & 169 & 67.9 & 87 & 74.4 & 82 & 62.1 \\
\hline yes $^{\mathrm{c}}$ & 80 & 32.1 & 30 & 25.6 & 50 & 37.9 \\
\hline based on moderate activity & 33 & 13.3 & 13 & 11.1 & 20 & 15.2 \\
\hline based on VV activity & 19 & 7.6 & 8 & 6.8 & 11 & 8.3 \\
\hline based on metabolic equivalent & 28 & 11.2 & 9 & 7.7 & 19 & 14.4 \\
\hline
\end{tabular}

Table 2. Physical activity parameters of study participants*. $95 \%$ CI, $95 \%$ confidence interval; cpm, counts per minute; GM, geometric mean; IQR, interquartile range; $\mathrm{min} / \mathrm{d}$, minutes per day; n, number; $\mathrm{PA}$, physical activity; VV, vigorous-to-very-vigorous; WHO, World Health Organization. *Activity intensities were determined based on triaxial $24 \mathrm{~h}$-accelerometry vector magnitude defining $0-78 \mathrm{cpm}$ as 'inactivity', $79-2,690 \mathrm{cpm}$ as 'low-intensity', 2,691-6,166 cpm as 'moderate', and $\geq 6,167 \mathrm{cpm}$ as VV activity ${ }^{27,28}$. ${ }^{\mathrm{a}}$ Meeting WHO PA recommendation' ('yes') was defined as accumulating $\geq 150 \mathrm{~min} /$ week or $\geq 75 \mathrm{~min} /$ week of vigorous activity/week (here: VV activity) (mean weekly estimates: mean min/d per participant multiplied by 7), spent in activity bouts $\geq 10$ minutes, or an equivalent combination of these ${ }^{1}$. For the latter metabolic equivalents of tasks (METs)/week were calculated, when multiplying mean weekly estimates in moderate and VV activity by 4 and 8 METs, respectively, as described before ${ }^{29}$. Achieving with the sum of both $\geq 450 \mathrm{METs} /$ week, this was classified as 'meeting WHO PA recommendation'. Not meeting any of the aforementioned criteria was classified as 'not meeting WHO recommendation'. Bold: $p$-value $<0.05$.

\section{Discussion}

The present analyses aimed to identify factors related to time in different PA intensities using multiday $24 \mathrm{~h}$-accelerometry in a population-based sample. We found that higher age and current compared to never smoking were associated with more time spent in low-intensity and less time spent in VV activity, while higher BMI was related to less time spent in low-intensity activity. Furthermore, having versus not having a university entrance qualification and no versus full time employment were both associated with more time spent in inactivity and less time in low-intensity activity. Finally, current compared to never smoking was associated with a lower likelihood of meeting the WHO PA recommendation. These mutually adjusted associations were further independent of sex, WC, alcohol consumption, net household income, marital status, diabetes mellitus, dyslipidaemia, and study centre, respectively. The identified factors should be taken into account when planning public health measures aiming to increase PA.

Higher age was related to less time in VV activity and more time in low-intensity activity. These findings are consistent with previous findings from cross-sectional and longitudinal studies, including $24 \mathrm{~h}$-accelerometry-based data $^{29,32-34}$. A changing engagement in activity intensities with age is plausible due to an age-related decline in cardiorespiratory and musculoskeletal fitness, thus, limiting resources to perform highly intense $\mathrm{PA}^{35,36}$. Furthermore, psychosocial barriers, like a decrease in social support for PA or feeling 'too old' for high intensity PA may contribute to the observed decline in VV activity and the simultaneous increase in low-intensity activity with higher age ${ }^{37}$. Although not modifiable, age as a PA-related factor seems to be relevant for activity intensity on the population level. Interestingly, age was not related to time in inactivity, indicating that although higher intense activity may decrease with age, $24 \mathrm{~h}$-accelerometry-based inactivity time may not vary significantly.

Additionally, higher BMI was associated with less time spent in low-intensity activity, which is in line with previous findings for accelerometry-based time in light activity ${ }^{38}$. This effect can probably partly be explained by the fact that BMI is strongly correlated with body weight ${ }^{39,40}$ and thus, that persons with higher BMI may more likely be less active (in terms of acceleration) due to the higher effort of movements; in turn, the activity-related energy expenditure does not compulsorily differ from persons with lower BMI ${ }^{41}$. Further, higher BMI is related to an impaired cardiopulmonary fitness that may limit the capacity of being active ${ }^{42}$, and to psychological traits, like a decreased self-efficacy, self-confidence, and perceived fitness that, in turn, are inversely related to $\mathrm{PA}^{9,13}$. Interestingly, although reported previously $y^{3,43-45}$, we did not find an association of BMI with other activity intensities, but there was a non-significant trend for a positive association with inactivity time. Although the effect size was small for a 1-unit increase in BMI, considering larger changes in this modifiable factor, our analyses indicate an epidemiologically relevant impact of BMI on time in low-intensity activity.

Further, compared to never smokers, current smokers spent less time in VV, while spending almost half an hour more time in low-intensity activity. These observations are plausible, given the negative impact of smoking on cardiopulmonary and musculoskeletal functioning and fitness, energy and oxygen metabolism and supply ${ }^{46,47}$. All of those result in a perceived higher effort, decreased capability, and earlier fatigue, when being active at 


\begin{tabular}{|c|c|c|c|c|c|c|c|c|c|c|c|c|}
\hline \multirow[b]{2}{*}{ Potential factors } & \multicolumn{3}{|c|}{ time in inactivity, $\mathrm{min} / \mathrm{d}$} & \multicolumn{3}{|c|}{ time in low-intensity activity, min/d } & \multicolumn{3}{|c|}{ time in moderate activity, $\mathrm{min} / \mathrm{d}$} & \multicolumn{3}{|c|}{ time in VV activity, $\mathrm{min} / \mathrm{d}$} \\
\hline & $\beta$ & $95 \% \mathrm{CI}$ & $\mathbf{p}$ & $\beta$ & 95\% CI & $\mathbf{p}$ & $\beta$ & $95 \% \mathrm{CI}$ & $\mathbf{p}$ & $\beta$ & $95 \% \mathrm{CI}$ & $\mathbf{p}$ \\
\hline sex (men vs. women) & 9.1 & $(-19.6,37.9)$ & 0.53 & -19.3 & $(-41.8,3.2)$ & 0.09 & 9.2 & $(-4.2,22.6)$ & 0.18 & 1.0 & $(-3.3,5.3)$ & 0.65 \\
\hline age ( 5 years) & -6.1 & $(-13.0,0.7)$ & 0.08 & 7.3 & $(2.0,12.7)$ & 0.007 & -0.4 & $(-2.8,2.1)$ & 0.77 & -0.8 & $(-1.4,-0.2)$ & 0.007 \\
\hline BMI, $\mathrm{kg} / \mathrm{m}^{2}$ & 3.9 & $(0.6,7.3)$ & 0.02 & -3.7 & $(-6.3,-1.2)$ & 0.005 & 0.1 & $(-1.4,1.5)$ & 0.93 & -0.3 & $(-0.6,0.1)$ & 0.12 \\
\hline $\mathrm{WC}, \mathrm{cm}^{\mathrm{a}}$ & 1.6 & $(-0.3,3.5)$ & 0.10 & -0.5 & $(-2.0,1.0)$ & 0.53 & -1.1 & $(-1.9,-0.2)$ & 0.02 & -0.1 & $(-0.3,0.1)$ & 0.43 \\
\hline smoking status & & & 0.13 & & & 0.0006 & & & 0.56 & & & 0.005 \\
\hline never & 0 & (reference) & & 0 & (reference) & & 0 & (reference) & & 0 & (reference) & \\
\hline current & -19.8 & $(-47.5,7.9)$ & & 29.2 & $(7.5,50.9)$ & & -5.5 & $(-16.5,5.5)$ & & -3.9 & $(-6.3,-1.5)$ & \\
\hline former & 9.0 & $(-12.2,30.3)$ & & -6.7 & $(-23.4,10.0)$ & & 0.1 & $(-8.4,8.6)$ & & -2.4 & $(-4.6,-0.3)$ & \\
\hline alcohol consumption & & & 0.24 & & & 0.55 & & & 0.10 & & & 0.15 \\
\hline never & -44.9 & $(-106.3,16.6)$ & & 16.4 & $(-33.7,66.4)$ & & 26.1 & $(-1.2,53.4)$ & & 2.4 & $(-1.8,6.7)$ & \\
\hline max. 1x/month & 0 & (reference) & & 0 & (reference) & & 0 & (reference) & & 0 & (reference) & \\
\hline $2-4 \mathrm{x} / \mathrm{month}$ & -4.0 & $(-32.7,24.6)$ & & 9.5 & $(-13.2,32.2)$ & & -3.4 & $(-15.1,8.3)$ & & -2.1 & $(-5.8,1.7)$ & \\
\hline $2-3 x /$ week & -13.3 & $(-44.1,17.5)$ & & 13.1 & $(-11.9,38.0)$ & & 0.9 & $(-10.5,12.3)$ & & -0.7 & $(-4.7,3.3)$ & \\
\hline$\geq 4 \mathrm{x} /$ week & -32.4 & $(-66.3,1.6)$ & & 23.2 & $(-3.8,50.2)$ & & 10.3 & $(-4.0,24.5)$ & & -1.1 & $(-5,2.8)$ & \\
\hline $\begin{array}{l}\text { university entrance qualification } \\
\text { (yes vs. no) }\end{array}$ & 35.9 & $(13.0,58.8)$ & 0.002 & -31.7 & $(-49.9,-13.4)$ & 0.0008 & -4.7 & $(-13.0,3.5)$ & 0.26 & 0.5 & $(-1.7,2.7)$ & 0.66 \\
\hline employment status & & & $<0.0001$ & & & 0.0001 & & & 0.44 & & & 0.63 \\
\hline full time & 0 & (reference) & & 0 & (reference) & & 0 & (reference) & & 0 & (reference) & \\
\hline part time & -3.4 & $(-29.2,22.4)$ & & 3.5 & $(-17.1,24.1)$ & & 1.0 & $(-9.2,11.3)$ & & -1.1 & $(-4.0,1.8)$ & \\
\hline not employed & 66.2 & $(34.7,97.7)$ & & -50.7 & $(-76.6,-24.8)$ & & -14.1 & $(-26.3,-1.8)$ & & -1.4 & $(-4.4,1.6)$ & \\
\hline net household income per month & & & 0.64 & & & 0.85 & & & 0.17 & & & 0.60 \\
\hline$<2,500 €$ & 0 & (reference) & & 0 & (reference) & & 0 & (reference) & & 0 & (reference) & \\
\hline $2,500-4,000 €$ & 6.8 & $(-16.7,30.4)$ & & -6.4 & $(-23.4,10.6)$ & & -0.5 & $(-11.0,10.1)$ & & 0.1 & $(-2.1,2.2)$ & \\
\hline$>4,000 €$ & 11.8 & $(-18.8,42.5)$ & & -7.2 & $(-30.7,16.3)$ & & -4.8 & $(-16.3,6.7)$ & & 0.1 & $(-3.2,3.5)$ & \\
\hline n. a. & -15.6 & $(-56.0,24.8)$ & & -7.8 & $(-36.9,21.3)$ & & 20.4 & $(-1.5,42.3)$ & & 3.0 & $(-1.5,7.5)$ & \\
\hline $\begin{array}{l}\text { marital status (married, no vs. } \\
\text { yes) }\end{array}$ & 21.3 & $(-3.7,46.3)$ & 0.09 & -12.1 & $(-32.6,8.3)$ & 0.24 & -6.3 & $(-15.5,2.9)$ & 0.18 & -2.9 & $(-5.7,-0.2)$ & 0.04 \\
\hline diabetes mellitus (yes vs. no) & -18.3 & $(-72.7,36.1)$ & 0.51 & 12.3 & $(-26.6,51.2)$ & 0.53 & 5.3 & $(-13.3,23.8)$ & 0.58 & 0.7 & $(-1.9,3.4)$ & 0.59 \\
\hline dyslipidaemia (yes vs. no) & -2.6 & $(-28.1,22.9)$ & 0.84 & 1.6 & $(-19.2,22.4)$ & 0.88 & 0.4 & $(-8.6,9.4)$ & 0.93 & 0.6 & $(-1.2,2.3)$ & 0.51 \\
\hline
\end{tabular}

Table 3. Multivariable association of physical activity-related factors and time in different activity intensities*, total $(\mathrm{N}=249)$. Information was derived from self-reports during a personal interview, anthropometric measures were taken by trained personnel 95\% CI, 95\% confidence interval; BMI, body mass index; min/d, minutes per day; n. a., not available; vs., versus; VV, vigorous to very vigorous; $\mathrm{WC}$, waist circumference. ${ }^{*}$ Results were derived from four different multivariable linear regression analyses with factors potentially related to physical activity included as independent and time spent in the four different activity intensities included as single dependent variable. $\beta$-coefficients can be interpreted as absolute change in time in the different activity intensities in minutes per day, referring to a 1-unit increase for continuous variables or to the respective reference category for categorical variables. Model includes sex, age, body mass index (BMI), waist circumference (residually adjusted for BMI), smoking status, alcohol consumption, university entrance qualification, employment status, net household income, marital status, diabetes, dyslipidaemia, and study centre. Activity intensities were determined based on triaxial $24 \mathrm{~h}$-accelerometry vector magnitude defining $0-78 \mathrm{cpm}$ as 'inactivity', 79-2,690 cpm as 'low-intensity', 2,691-6,166 cpm as 'moderate', and $\geq 6,167 \mathrm{cpm}$ as VV activity ${ }^{27,28}$. ${ }^{a}$ Residually adjusted for BMI. Bold: statistically significant when accounting for multiple testing ( $p$ value $<0.01)$.

higher intensity ${ }^{46,47}$, and may, thus, deter smokers from higher intensity activity. Additionally, smoking is often related to a generally less health-conscious lifestyle, including lower sports and exercise engagement ${ }^{48}$, i.e., behaviours of higher intensity. Finally, smokers tend to have a higher discount rate than never smokers, i.e. a higher behavioural impatience and devaluation of delayed outcomes, indicating that they favour an immediate reward (ease of low-intensity activity) even when it is less advantageous above a larger reward being beneficial at a later time (better health ${ }^{49,50}$, making a preference for low over higher intensity activities reasonable. A previous longitudinal study showed that smoking versus non-smoking is associated with a lower likelihood of being persistently moderately or vigorously active over a 10 -years period ${ }^{32}$. Thus, one may conclude that smoking has a long-term effect on intensity of individual PA. This assumption is supported by our observation that former compared to never smokers tended to spent less time in VV activity; however, this association was not statistically significant.

Systematic analyses of reviews and meta-analyses suggest that socio-economic indicators are associated with $\mathrm{PA}^{6,34,51}$. We found two socio-economic factors to be related to time spent in inactivity. Firstly, having a university entrance qualification versus no such qualification was associated with more than half an hour more inactivity time, 


\begin{tabular}{|c|c|c|c|c|}
\hline \multirow{3}{*}{$\begin{array}{l}\text { potential factors } \\
\text { sex (men vs. women) }\end{array}$} & \multicolumn{4}{|c|}{ meeting WHO PA recommendation ${ }^{a}$ (yes vs. no) } \\
\hline & \multirow{2}{*}{$\begin{array}{l}\text { OR } \\
0.95\end{array}$} & \multirow{2}{*}{\begin{tabular}{|l}
$\mathbf{9 5} \% \mathrm{CI}$ \\
$(0.35,2.53)$
\end{tabular}} & \multicolumn{2}{|c|}{$\mathbf{p}$} \\
\hline & & & 0.91 & \\
\hline age ( 5 years) & 1.09 & $(0.89,1.32)$ & 0.41 & \\
\hline BMI, $\mathrm{kg} / \mathrm{m}^{2}$ & 0.96 & $(0.87,1.05)$ & 0.36 & \\
\hline $\mathrm{WC}, \mathrm{cm}^{\mathrm{b}}$ & 0.97 & $(0.91,1.04)$ & 0.42 & \\
\hline smoking status & & & & 0.004 \\
\hline never & 1 & (reference) & & \\
\hline current & 0.21 & $(0.08,0.53)$ & 0.001 & \\
\hline former & 0.59 & $(0.30,1.16)$ & 0.12 & \\
\hline alcohol consumption & & & & 0.68 \\
\hline never & 0.32 & $(0.02,4.72)$ & 0.41 & \\
\hline maximal $1 \times /$ month & 1 & (reference) & & \\
\hline $2-4 \times /$ month & 1.51 & $(0.62,3.69)$ & 0.36 & \\
\hline $2-3 \times /$ week & 1.59 & $(0.62,4.09)$ & 0.34 & \\
\hline$\geq 4 \times /$ week & 1.30 & $(0.44,3.87)$ & 0.64 & \\
\hline university entrance qualification (yes vs. no) & 1.29 & $(0.66,2.51)$ & 0.46 & \\
\hline employment status & & & & 0.95 \\
\hline full time & 1 & (reference) & & \\
\hline part time & 0.89 & $(0.38,2.08)$ & 0.79 & \\
\hline not employed & 1.04 & $(0.38,2.85)$ & 0.94 & \\
\hline net household income per month & & & & 0.85 \\
\hline$<2,500 €$ & 1 & (reference) & & \\
\hline $2,500-4,000 €$ & 1.09 & $(0.49,2.43)$ & 0.83 & \\
\hline$>4,000 €$ & 0.86 & $(0.34,2.17)$ & 0.75 & \\
\hline n. a. & 1.67 & $(0.39,7.09)$ & 0.49 & \\
\hline marital status (married, no vs. yes) & 1.67 & $(0.77,3.62)$ & 0.20 & \\
\hline diabetes mellitus (yes vs. no) & 0.58 & $(0.15,2.28)$ & 0.44 & \\
\hline dyslipidaemia (yes vs. no) & 0.96 & $(0.44,2.11)$ & 0.93 & \\
\hline
\end{tabular}

Table 4. Multivariable association of physical activity-related factors and fulfilment of WHO physical activity recommendation*, total $(\mathrm{N}=249)$. Information was derived from self-reports during a personal interview, anthropometric measures were taken by trained personnel 95\% CI, 95\% confidence interval; BMI, body mass index; n. a., not available; OR, odds ratio; PA, physical activity; vs., versus; WC, waist circumference; WHO, World Health Organization. *Results were derived from a multivariable logistic regression analysis with factors potentially related to physical activity included as independent and fulfilment of the World Health Organization (WHO) physical activity recommendation included as dependent variable. $\beta$-coefficients can be interpreted as change in the likelihood (odds ratio, OR) of meeting the WHO recommendation, referring to a 1-unit increase for continuous variables or to the respective reference category for categorical variables. Model includes sex, age, body mass index (BMI), waist circumference (residually adjusted for BMI), smoking status, alcohol consumption, university entrance qualification, employment status, net household income, marital status, diabetes,

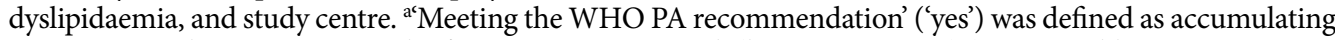
$\geq 150 \mathrm{~min} /$ week or $\geq 75 \mathrm{~min} /$ week of vigorous activity/week (here: VV activity) (mean weekly estimates: mean $\mathrm{min} / \mathrm{d}$ per participant multiplied by 7 ), spent in activity bouts $\geq 10$ minutes, or an equivalent combination of these $^{1}$. For the latter, metabolic equivalents of tasks (METs)/week were calculated, when multiplying mean weekly estimates in moderate and VV activity by 4 and $8 \mathrm{METs}$, respectively, as described before ${ }^{29}$. Achieving with the sum of both $\geq 450 \mathrm{METs} /$ week, this was classified as 'meeting WHO PA recommendation'. Not meeting any of the aforementioned criteria was classified as 'not meeting WHO recommendation'. ' Residually adjusted for BMI. Bold: statistically significant when accounting for multiple testing ( $p$-value $<0.01)$.

coming at the expense of time in low-intensity activity. This may be surprising, given most previous studies showing higher education to be related to higher self-reported PA levels ${ }^{6,34,51}$. Persons with versus without a university entrance qualification may engage in more targeted activities, like sports, due to more financial resources and a better knowledge of PA benefits. However, on the other hand, they may more likely have less PA-demanding jobs, e.g., white-collar work, while low occupational PA-levels, in turn, are related to low leisure-time PA levels ${ }^{52}$. Further, we did not rely on self-reported activities, as in most previous studies, but used $24 \mathrm{~h}$-accelerometry and, thus, continuously measured overall movement across the day. These reasons may explain our findings of a shift from active time at low intensity to more inactivity time when having versus not having a university entrance qualification.

Similarly, no versus full time employment was related to more than one hour more time in inactivity, with this plus coming at the expense of time spent in low-intensity activity. These associations were the strongest observed in the present analyses and are in line with previous studies on self-reported $\mathrm{PA}^{34,51}$. A shift from low-intensity to time in inactivity is plausible for unemployment versus full time employment, as it can partly be explained by a lack of transportation (way to work) or occupational PA that may account for a large proportion of habitual PA, 
especially in manual, blue-collar work ${ }^{52}$. Furthermore, not being employed is associated with the risk of social deprivation, lacking interest in PA, as well as with poorer health and well-being, being, in turn, all related to lower PA engagement ${ }^{13,33,53}$. Therefore, populations with high levels of unemployment, but also higher education and sedentary jobs may represent targets for PA promotion strategies.

Our linear regression analyses include any time of PA spent in the respective PA parameter. The WHO in their definition recommends that these activities should make up at least $150 \mathrm{~min} /$ week of moderate or $75 \mathrm{~min} /$ week of VV activity spent in bouts of at least 10 minutes. When applying these criteria, we found that current compared to never smoking was associated with a decreased likelihood of meeting the WHO recommendation by $79 \%$. Due to the relevant impact (mean difference in time in activity intensities and OR) and consistency across main and sensitivity analyses, smoking is considered to be an important public health-related lifestyle risk factor associated with PA. In their 2018 edition of PA guidelines for adults, the U.S. Department of Health and Human Services removed the 10-min bouts requirement ${ }^{54}$. In our sample, all participants fulfilled the PA recommendations when based on these US guidelines.

In our analyses, there was a tendency for a sex difference in the associations of WC and smoking with moderate activity and with the likelihood of meeting the WHO PA recommendation, as well as of net household income with inactivity time. However, when accounting for multiple testing, these differences were not statistically significant. Further studies with larger sample sizes are warranted to assess whether there are true differences between sexes.

Most previous studies used uniaxial accelerometry during waking to assess $\mathrm{PA}^{16-19}$, whereas newer cohorts like the NAKO or UK Biobank use triaxial $24 \mathrm{~h}$-accelerometry ${ }^{20-22}$. While the advantage of triaxial outputs is debated $^{55}$, previous studies did not find substantial differences between uni- and triaxial measures regarding their association with energy expenditure, rather, there was a trend for slightly stronger associations and a more precise PA assessment based on triaxial measures ${ }^{56-59}$. In separate previous analyses, we also did not find significant differences in the association of uni- and triaxial outputs with the activity-related energy expenditure measured by doubly-labelled water. In our present study, we, in line with new epidemiologic studies, rely on triaxial $24 \mathrm{~h}$-accelerometry, expecting that this allows a more comprehensive PA assessment, while accepting limited comparability of our findings.

A strength of our study was the focus on multiday triaxial $24 \mathrm{~h}$-accelerometry to assess PA in a population-based observational study, thus allowing for a more comprehensive and objective PA assessment than in previous studies using self-reported PA or uniaxial accelerometry during waking only ${ }^{18,60}$. The multicentric study design and application of standard protocols allowed collecting a broad spectrum of participants' characteristics across Germany with identical methods, thus permitting joint analyses of data. We found no substantial differences between included and excluded participants. Nevertheless, the sample was limited to adults and a selection bias cannot be ruled out. It is well known that participants in epidemiologic studies tend to be more health-conscious than the general population ${ }^{61}$. Findings on PA-related factors may be different in other populations (e.g., diseased populations or other age ranges) and further studies are warranted to investigate PA-related factors in diverse populations. Although participants were asked to pursue their daily routine, study participation and use of accelerometry may affect PA behaviour. However, in a previous study, we did not find any indications of behavioural bias regarding PA when using multiday $24 \mathrm{~h}$-accelerometry ${ }^{27}$. Except for anthropometry, factors investigated were self-reported; therefore, social desirability and reporting bias cannot be ruled out. However, if any, we assume such effects to be randomly distributed across PA levels and, thus, to be negligible. Likewise, although we adjusted for a number of variables, residual confounding cannot be ruled out. A further limitation may be that accelerometer data does not entail the domain of PA, such as leisure-time or occupational PA. However, we considered total duration and intensity of PA over $24 \mathrm{~h}$ to be the most relevant factor in terms of affecting health. For activity intensity determination, we used a modified version of the 'Freedson Adult VM3 (2011)' algorithm, but we were not able to separate sedentary behaviour or sleeping times, which are, however, of epidemiologic interest and are encompassed in 'time in inactivity ${ }^{62-64}$. Additionally, since we had no information on accelerometer non-wear times and wake-sleep-rhythms, we used a conservative approach assuming the waking period between $6 \mathrm{AM}$ and $10 \mathrm{PM}$ not allowing for 120 non-wear minutes during this period ${ }^{26}$. However, this waking period may not hold true for all participants, and sleeping during this period may have caused unnecessary exclusion of participants due to falsely detected non-wear time based on accelerometry. If any, this may have limited the power of our analyses, but findings should not have been substantially affected. Our analyses may further include non-wear time periods $<120$ minutes, which may false-positively be assigned to time in inactivity, when participants were physically active while not wearing the accelerometer ${ }^{26}$. However, results did not substantially change when we used a non-wear time algorithm requiring periods $>60$ instead of $>120$ minutes. Compared to previous studies using uniaxial accelerometry during waking, time in moderate and VV activity, and, thus, the proportion of participants meeting the WHO recommendation were slightly higher in our population ${ }^{29,60}$. However, we used triaxial-derived and, thus, other cut points to determine PA intensities than previous studies, and triaxial $24 \mathrm{~h}$-accelerometry, making a more comprehensive PA assessment and higher PA levels plausible. This was already observed in our previous study using the same approach ${ }^{27}$. However, we do not expect findings on PA-related factors to be substantially affected by slightly higher group PA levels. It is important to note that our study sample was relatively small and analyses were cross-sectional, thus precluding causal inference, and prospective studies are warranted to further investigate PA-related factors. The comprehensive study protocol and large sample size of the NAKO will provide data for repeating our analyses on a large scale as well as for sound longitudinal analyses on a wide range of determinants and correlates of $24 \mathrm{~h}$-accelerometry-based $\mathrm{PA}^{20,21,25}$. Finally, it should be noted that non-significant results in our analyses may not inevitably indicate the absence of a true association, but may also be due to the limited sample size.

In conclusion, using multiday $24 \mathrm{~h}$-accelerometry to assess habitual PA in a population-based study, our results suggest that the assessed biological, behavioural, socio-economic and socio-cultural factors show 
distinct associations with $24 \mathrm{~h}$-accelerometry-based time in activity intensities. Our findings underpin the relevance of times in different intensities for individual habitual PA, being comprehensively assessable using $24 \mathrm{~h}$-accelerometry. The identified modifiable factors may represent targets for public health measures of PA promotion strategies, while the observed unmodifiable PA-related factors allow identification of target subgroups for such programs.

\section{Data availability}

The data that support the findings of this study are available from the NAKO but restrictions apply to the availability of these data, which were used under license for the current study, and so are not publicly available. Data are however available from the authors upon reasonable request and with permission of the NAKO.

Received: 10 January 2019; Accepted: 22 December 2019;

Published online: 21 January 2020

\section{References}

1. World Health Organization. Global Recommendations on Physical Activity for Health. World Health Organization, Geneva. (2010). http://whqlibdoc.who.int/publications/2010/9789241599979_eng.pdf?ua=1. (accessed 4 July 2018).

2. World Health Organization. Global Health Risks - Mortality and burden of disease attributable to selected major risks. World Health Organization, Geneva. (2009). http://www.who.int/iris/handle/10665/44203. (accessed 22 July 2018).

3. Arem, H. et al. Leisure time physical activity and mortality: A detailed pooled analysis of the dose-response relationship. JAMA Intern Med. 175, 959-967 (2015).

4. World Health Organization. Global status report on noncommunicable diseases. World Health Organization, Geneva. (2014). http:// www.who.int/nmh/publications/ncd-status-report-2014/en/. (accessed 26 April 2018).

5. Sallis, J. F. et al. An ecological approach to creating active living communities. Annu Rev Public Health. 27, 297-322 (2006).

6. O'Donoghue, G. et al. Socio-economic determinants of physical activity across the life course: A "DEterminants of DIet and Physical ACtivity" (DEDIPAC) umbrella literature review. PLoS One. 13, e0190737 (2018).

7. Carlin, A. et al. A life course examination of the physical environmental determinants of physical activity behaviour: A "Determinants of Diet and Physical Activity" (DEDIPAC) umbrella systematic literature review. PLoS One. 12, e0182083 (2017).

8. Condello, G. et al. Behavioral determinants of physical activity across the life course: a "DEterminants of DIet and Physical ACtivity" (DEDIPAC) umbrella systematic literature review. Int J Behav Nutr Phys Act. 14, 58 (2017).

9. Cortis, C. et al. Psychological determinants of physical activity across the life course: A "DEterminants of DIet and Physical ACtivity" (DEDIPAC) umbrella systematic literature review. PLoS One. 12, e0182709 (2017).

10. Jaeschke, L. et al. Socio-cultural determinants of physical activity across the life course: a 'Determinants of Diet and Physical Activity' (DEDIPAC) umbrella systematic literature review. Int J Behav Nutr Phys Act. 14, 173 (2017).

11. Puggina, A. et al. Policy determinants of physical activity across the life course: a 'DEDIPAC' umbrella systematic literature review. Eur J Public Health. 28, 105-118 (2018).

12. Aleksovska, K. et al. Biological determinants of physical activity across the life course: a "Determinants of Diet and Physical Activity" (DEDIPAC) umbrella systematic literature review. Sports Med Open. 5, 2 (2019).

13. Choi, J., Lee, M., Lee, J.-K., Kang, D. \& Choi, J.-Y. Correlates associated with participation in physical activity among adults: a systematic review of reviews and update. BMC Public Health. 17, 356 (2017).

14. Helmerhorst, H. J., Brage, S., Warren, J., Besson, H. \& Ekelund, U. A systematic review of reliability and objective criterion-related validity of physical activity questionnaires. Int J Behav Nutr Phys Act. 9, 103 (2012).

15. Samitz, G., Egger, M. \& Zwahlen, M. Domains of physical activity and all-cause mortality: systematic review and dose-response meta-analysis of cohort studies. Int J Epidemiol. 40, 1382-1400 (2011).

16. Bakrania, K. et al. Associations of mutually exclusive categories of physical activity and sedentary time with markers of cardiometabolic health in English adults: a cross-sectional analysis of the Health Survey for England. BMC Public Health. 16, 25-25 (2016).

17. Colley, R. C. et al. Physical activity of Canadian adults: accelerometer results from the 2007 to 2009 Canadian Health Measures Survey. Health rep. 22, 7-14 (2011).

18. Troiano, R. P. et al. Physical Activity in the United States Measured by Accelerometer. Med Sci Sports Exerc. 40, 181-188 (2008).

19. Van Domelen, D. R. et al. Employment and physical activity in the U.S. Am J Prev Med. 41, 136-145 (2011).

20. German National Cohort Consortium. The German National Cohort: aims, study design and organization. Eur J Epidemiol. 29, 371-382 (2014).

21. The German National Cohort - A prospective epidemiological study resource for health and disease research in Germany. The German National Cohort (2015). https://nako.de/wp-content/uploads/2015/07/Wissenschaftliches-Konzept-der-NAKO2.pdf. (accessed 4 April 2018).

22. Doherty, A. et al. Large Scale Population Assessment of Physical Activity Using Wrist Worn Accelerometers: The UK Biobank Study. PLoS One. 12, e0169649-e0169649 (2017).

23. Tudor-Locke, C., Camhi, S. M. \& Troiano, R. P. A catalog of rules, variables, and definitions applied to accelerometer data in the National Health and Nutrition Examination Survey, 2003-2006. Prev Chronic Dis. 9, E113 (2012).

24. Wijndaele, K. et al. Utilization and Harmonization of Adult Accelerometry Data: Review and Expert Consensus. Med Sci Sports Exerc. 47, 2129-2139 (2015).

25. Wichmann, H. E. et al. The German National Cohort. Bundesgesundheitsblatt Gesundheitsforschung Gesundheitsschutz. 55, 781-787 (2012).

26. Jaeschke, L. et al. $24 \mathrm{~h}$-accelerometry in epidemiological studies: automated detection of non-wear time in comparison to diary information. Sci Rep. 7, 2227 (2017).

27. Jaeschke, L., Steinbrecher, A., Jeran, S., Konigorski, S. \& Pischon, T. Variability and reliability study of overall physical activity and activity intensity levels using $24 \mathrm{~h}$-accelerometry-assessed data. BMC Public Health. 18, 530 (2018).

28. Sasaki, J. E., John, D. \& Freedson, P. S. Validation and comparison of ActiGraph activity monitors. J Sci Med Sport. 14, 411-416 (2011).

29. Hallal, P. C. et al. Global physical activity levels: surveillance progress, pitfalls, and prospects. Lancet. 380, 247-257 (2012).

30. White, H. A heteroskedasticity-consistent covariance matrix estimator and a direct test for heteroskedasticity. Econometrica. 48, $817-838$ (1980).

31. Freedson, P. S., Melanson, E. \& Sirard, J. Calibration of the Computer Science and Applications, Inc. accelerometer. Med Sci Sports Exerc. 30, 777-781 (1998).

32. Smith, L., Gardner, B., Fisher, A. \& Hamer, M. Patterns and correlates of physical activity behaviour over 10 years in older adults: prospective analyses from the English Longitudinal Study of Ageing. BMJ Open. 5, e007423 (2015). 
33. Luzak, A. et al. Physical activity levels, duration pattern and adherence to WHO recommendations in German adults. PLoS One. 12, e0172503 (2017).

34. European Commission. Special Eurobarometer 412/Wave EB80.2 'Sport and physical activity'. European Commission. (2014). http://ec.europa.eu/health/nutrition_physical_activity/docs/ebs_412_en.pdf. (accessed 4 April 2018).

35. Goldspink, D. F. Ageing and activity: their effects on the functional reserve capacities of the heart and vascular smooth and skeletal muscles. Ergonomics. 48, 1334-1351 (2005).

36. Lowery, E. M., Brubaker, A. L., Kuhlmann, E. \& Kovacs, E. J. The aging lung. Clin Interv Aging. 8, 1489-1496 (2013).

37. Lindsay Smith, G., Banting, L., Eime, R., O'Sullivan, G. \& van Uffelen, J. G. Z. The association between social support and physical activity in older adults: a systematic review. Int J Behav Nutr Phys Act. 14, 56 (2017).

38. Bann, D. et al. Light Intensity physical activity and sedentary behavior in relation to body mass index and grip strength in older adults: cross-sectional findings from the Lifestyle Interventions and Independence for Elders (LIFE) study. PLoS One. 10, e0116058-e0116058 (2015)

39. World Health Organization. Obesity: preventing and managing the global epidemic. Report of a WHO consultation. World Health Organ Tech Rep Ser. 894(i-xii), 1-253 (2000).

40. World Health Organization. Waist Circumference and Waist-Hip Ratio. Report of a WHO Expert Consultation. Geneva, 8-11 December 2008. World Health Organization, Geneva. (2011). http://www.who.int/nutrition/publications/obesity/WHO_report_ waistcircumference_and_waisthip_ratio/en/. (accessed 25 August 2018).

41. Westerterp, K. R. Physical activity and physical activity induced energy expenditure in humans: measurement, determinants, and effects. Front Physiol. 4, 90 (2013).

42. Lakoski, S. G. et al. Impact of Body Mass Index, Physical Activity, and Other Clinical Factors on Cardiorespiratory Fitness (from the Cooper Center Longitudinal Study). Am J Cardiol. 108, 34-39 (2011)

43. Allen, M. S. \& Vella, S. A. Longitudinal determinants of walking, moderate, and vigorous physical activity in Australian adults. Prev Med. 78, 101-104 (2015).

44. Besson, H. et al. A cross-sectional analysis of physical activity and obesity indicators in European participants of the EPICPANACEA study. Int J Obes. 33, 497-506 (2009).

45. Du, H. et al. Physical activity and sedentary leisure time and their associations with BMI, waist circumference, and percentage body fat in 0.5 million adults: the China Kadoorie Biobank study. Am J Clin Nutr. 97, 487-496 (2013).

46. Isabel, U. et al. Smoking habit, respiratory symptoms and lung function in young adults. Eur J Public Health. 15, 160-165 (2005).

47. Bernaards, C. M., Twisk, J. W., Van Mechelen, W., Snel, J. \& Kemper, H. C. A longitudinal study on smoking in relationship to fitness and heart rate response. Med Sci Sports Exerc. 35, 793-800 (2003).

48. Spring, B., Moller, A. C. \& Coons, M. J. Multiple health behaviours: overview and implications. J Public Health (Oxf). 34, i3-i10 (2012).

49. Story, G. W., Vlaev, I., Seymour, B., Darzi, A. \& Dolan, R. J. Does temporal discounting explain unhealthy behavior? A systematic review and reinforcement learning perspective. Front Behav Neurosci. 8, 76 (2014)

50. Chabris, C. F., Laibson, D., Morris, C. L., Schuldt, J. P. \& Taubinsky, D. Individual laboratory-measured discount rates predict field behavior. J Risk Uncertain. 37, 237 (2008).

51. Trost, S. G., Owen, N., Bauman, A. E., Sallis, J. F. \& Brown, W. Correlates of adults' participation in physical activity: review and update. Med Sci Sports Exerc. 34, 1996-2001 (2002).

52. Prince, S. A., Reed, J. L., McFetridge, C., Tremblay, M. S. \& Reid, R. D. Correlates of sedentary behaviour in adults: a systematic review. Obes Rev. 18, 915-935 (2017).

53. Social determinants of health: the solid facts. World Health Organization, Denmark. (2003). http://apps.who.int/iris/ bitstream/10665/108082/1/e59555.pdf. (accessed 26 July 2018).

54. U.S. Department of Health and Human Services. Physical Activity Guidelines for Americans, 2nd edition. Washington, DC: U.S. Department of Health and Human Services. (2018). https://health.gov/paguidelines/second-edition/pdf/Physical_Activity_ Guidelines_2nd_edition.pdf (accessed 8 May 2019).

55. Matthew, C. E. Calibration of accelerometer output for adults. Med Sci Sports Exerc. 37, S512-522 (2005).

56. Chen, K. Y. \& Bassett, D. R. Jr. The technology of accelerometry-based activity monitors: current and future. Med Sci Sports Exerc. 37, S490-500 (2005).

57. Shephard, R. J. The Objective Monitoring of Physical Activity. Prog Prev Med. 2, e0007 (2017).

58. Hills, A. P., Mokhtar, N. \& Byrne, N. M. Assessment of physical activity and energy expenditure: an overview of objective measures. Front Nutr. 1, 5 (2014).

59. Jeran, S., Steinbrecher, A. \& Pischon, T. Prediction of activity-related energy expenditure using accelerometer-derived physical activity under free-living conditions: a systematic review. Int J Obes (Lond). 40, 1187-1197 (2016).

60. Keevil, V. L. et al. Objective Sedentary Time, Moderate-to-Vigorous Physical Activity, and Physical Capability in a British Cohort. Med Sci Sports Exerc. 48, 421-429 (2016).

61. Ahrens, W. \& Pigeot, I. Handbook of epidemiology. Berlin Heidelberg: (Springer, 2005).

62. Biswas, A. et al. Sedentary time and its association with risk for disease incidence, mortality, and hospitalization in adults: a systematic review and meta-analysis. Ann Intern Med. 162, 123-132 (2015).

63. Cappuccio, F. P. \& Miller, M. A. Sleep and Cardio-Metabolic Disease. Curr Cardiol Rep. 19, 110 (2017).

64. Luyster, F. S., Strollo, P. J. Jr., Zee, P. C. \& Walsh, J. K. Sleep: A Health Imperative. Sleep. 35, 727-734 (2012).

\section{Acknowledgements}

The authors thank all study participants and the study personnel. The pretests for the NAKO study were funded by the Federal Ministry of Education and Research (Bundesministerium für Bildung und Forschung, Förderkennzeichen 01ER1001A-I), the states of Germany, and the Helmholtz Association.

\section{Author contributions}

L.J., A.S. and T.P. have directly participated in the data preparation, analysis, and interpretation of this manuscript. L.J. drafted the manuscript. W.H. and R.K. are P.I. of the central data management of the NAKO study and its pretest studies. All authors contributed to collecting the data, provided critical comments to the manuscript, and revised the text. All authors of this research paper have read and approved the final version submitted.

\section{Competing interests}

The authors declare no competing interests.

\section{Additional information}

Supplementary information is available for this paper at https://doi.org/10.1038/s41598-020-57648-w. 
Correspondence and requests for materials should be addressed to L.J.

Reprints and permissions information is available at www.nature.com/reprints.

Publisher's note Springer Nature remains neutral with regard to jurisdictional claims in published maps and institutional affiliations.

(c) Open Access This article is licensed under a Creative Commons Attribution 4.0 International License, which permits use, sharing, adaptation, distribution and reproduction in any medium or format, as long as you give appropriate credit to the original author(s) and the source, provide a link to the Creative Commons license, and indicate if changes were made. The images or other third party material in this article are included in the article's Creative Commons license, unless indicated otherwise in a credit line to the material. If material is not included in the article's Creative Commons license and your intended use is not permitted by statutory regulation or exceeds the permitted use, you will need to obtain permission directly from the copyright holder. To view a copy of this license, visit http://creativecommons.org/licenses/by/4.0/.

(C) The Author(s) 2020 